\title{
Restricciones de capacidad, exportación e I+D: Un análisis empírico con datos de empresas manufactureras españolas
}

\author{
JESÚS A. MUÑOZ-SEPÚLVEDA \\ Universidad Europea de Madrid y GRIPICO, c/ Tajo, s/n, 28670 Villaviciosa de Odón (Madrid), \\ España.E-mail: jesus.munoz@universidadeuropea.es
}

\begin{abstract}
RESUMEN
Los nuevos modelos económicos que incorporan heterogeneidad a nivel empresa asumen que las decisiones de producción son completamente flexibles, dejando de lado el potencial papel crítico que podrían desempeñar las restricciones de capacidad en las principales decisiones estratégicas de las empresas. Utilizando los datos de una muestra representativa de empresas manufactureras españolas, la Encuesta Sobre Estrategias Empresariales (ESEE), este artículo estima un modelo Probit bivariado para evaluar el impacto de las restricciones físicas de capacidad sobre la decisión conjunta de exportar e invertir en I+D. El principal resultado del trabajo indica que las empresas restringidas son menos propensas a participar en estas estrategias.
\end{abstract}

Palabras clave: Exportaciones, I+D, restricciones de capacidad, probit bivariado.

\section{Capacity Constraints, Exports and R\&D: An Empirical Analysis with Data of Spanish Manufacturing Firms}

\begin{abstract}
Most of the new models based on firm heterogeneity assume that production is completely flexible and do not take into account the critical role of capacity constraints in firm's strategic decisions. However, it seems clear that capacity-constrained firms cannot freely increase production. By using data drawn from the Encuesta Sobre Estrategias Empresariales (ESEE) about Spanish manufacturing firms, this article applies a bivariate probit model to empirically estimate the effect of physical capacity constraints in the firm's joint decision to export and invest in $\mathrm{R} \& \mathrm{D}$. The main result of the article reveals that firms facing capacity constraints are less prone to engage in these strategies.
\end{abstract}

Keywords: Export, R\&D, Capacity Constraints, Bivariate Probit.

Clasificación JEL: F10, F14, O30

Artículo recibido en octubre de 2016 y aceptado en diciembre de 2016

Artículo disponible en versión electrónica en la página www.revista-eea.net, ref. ə-35103 


\section{INTRODUCCIÓN}

Los nuevos modelos con empresas heterogéneas que analizan las decisiones estratégicas empresariales más relevantes, comenzando por Melitz (2003), han abordado ampliamente los principales factores que influyen en las estrategias de exportación e innovación. En general, todos estos modelos concluyen que son fundamentalmente las características de las empresas -incluyendo, entre otras, el tamaño, la productividad, la edad o el nivel de salarios- las principales variables que afectan de forma significativa sobre la probabilidad de llevar a cabo estas actividades. ${ }^{1}$ Estos modelos omiten, sin embargo, un elemento potencialmente importante que podría condicionar dicho comportamiento estratégico. Específicamente, asumen que las decisiones de producción son plenamente flexibles, dejando de lado el potencial papel crítico que podrían desempeñar las restricciones de capacidad productiva.

La reciente crisis económica y financiera ha generado un incremento de la literatura que considera el nivel de utilización de la capacidad productiva y las expectativas de demanda como unos importantes determinantes en las estrategias de exportación e inversión en I+D. En general, estos estudios asumen que la existencia de restricciones de capacidad modifica la estructura de costes marginales de la empresa, siendo estos crecientes en las empresas más restringidas (Ahn y McQuoid, 2012; Blum et al., 2013). Parece evidente, por tanto, que esta nueva estructura de costes marginales podría condicionar el desarrollo de las actividades de exportación e innovación. Por un lado, respecto a la estrategia de exportación, las empresas restringidas se encuentran produciendo a plena capacidad y no son capaces de incrementar más su producción para abastecer también a los mercados exteriores. ${ }^{2}$ Así, el proceso de ajuste entre los niveles potenciales de demanda y los inputs fijos (principalmente capital) dependería de la utilización de la capacidad productiva de la empresa.

Por otro lado, y respecto a la estrategia de I+D, las empresas suelen innovar para alcanzar beneficios indirectos relacionados con una mayor percepción por parte de los consumidores o para conseguir una mayor flexibilidad y adaptación frente a los costes y shocks de demanda. De esta forma, las empresas que se enfrentan a restricciones de capacidad contarían con potenciales dificultades para enfrentarse a estos shocks de demanda, lo que podría influir negativamente en el desarrollo de estrategias de I+D. Considerando lo expuesto anteriormente,

\footnotetext{
${ }^{1}$ Ver Bernard et al. (2003), Helpman et al. (2004), Melitz y Ottaviano (2008) o Lileeva y Trefler (2010) para más detalles.

${ }^{2}$ En este sentido, es necesario destacar una nueva rama de la literatura que sugiere la existencia de una relación de sustitución entre las ventas domésticas y de exportación (Ahn y McQuoid, 2012; Muñoz-Sepúlveda, 2014). Dicha relación podría implicar, por tanto, que las empresas que se enfrentan a restricciones de capacidad tienden a reducir sus ventas domésticas para poder entrar en nuevos mercados exteriores.
} 
este trabajo pretende analizar cuál es el efecto de las restricciones de capacidad sobre la realización de actividades de exportación e I+D.

Para llevar a cabo el análisis empírico se han utilizado los datos a nivel de empresa de la Encuesta Sobre Estrategias Empresariales (ESEE), elaborada por la Fundación SEPI y el Ministerio de Industria, Turismo y Comercio. El análisis econométrico estima un modelo Probit bivariado que permite evaluar la influencia de las restricciones de capacidad (y de otras variables relevantes a nivel de empresa) sobre la probabilidad conjunta de exportar e innovar. En este sentido, y considerando la tasa de utilización de la capacidad de las empresas, se utilizan tres indicadores o umbrales distintos para medir la existencia de restricciones de capacidad. Específicamente, se considera que una empresa está restringida cuando su tasa de utilización es (i) superior al 95\%, (ii) igual al 100\% o (iii) superior a un umbral específico calculado para cada industria y año. ${ }^{3} \mathrm{La}$ principal hipótesis que se desea contrastar es si las empresas con restricciones de capacidad son menos propensas a exportar o a invertir en I+D.

Los resultados empíricos sugieren que la tasa de utilización de la capacidad y la existencia de restricciones en la capacidad productiva juegan un papel esencial en la participación en estas decisiones estratégicas. Por un lado, los resultados revelan que una alta tasa de utilización de la capacidad en el periodo previo incrementa la probabilidad conjunta de exportar y realizar I+D. Por otro lado, los resultados señalan que la existencia de restricciones en la capacidad disminuye considerablemente la probabilidad de llevar a cabo dichas estrategias.

El artículo se organiza como sigue. La siguiente sección realiza una revisión de la reciente literatura que ha analizado el papel de las restricciones de capacidad físicas y financieras. La sección 3 describe la base de datos utilizada y presenta algunos resultados descriptivos importantes. A continuación, la sección 4 presenta el análisis econométrico y los principales resultados obtenidos. Por último, la sección 5 resume las conclusiones más relevantes.

\section{LITERATURA PREVIA}

La literatura que ha analizado los efectos de las restricciones de capacidad en las empresas se ha incrementado significativamente en la última década. En general, estos estudios asumen que los factores productivos (principalmente aquellos relacionados con el capital) se ajustan de forma gradual hasta alcanzar el nivel de equilibrio a largo plazo, enfatizando el papel de las restricciones de capacidad (físicas y financieras) en las decisiones estratégicas óptimas de las empresas. Dicho de otra forma, las restricciones de capacidad podrían vincularse con la presencia de un proceso lento de ajuste entre los inputs y el nivel de

\footnotetext{
${ }^{3}$ Ver Smolny (2003), Ahn y McQuoid (2012) y Crespo y Muñoz-Sepúlveda (2015) para más detalles.
} 
demanda. Adicionalmente, esta literatura también sugiere un cambio potencial en la estructura de costes marginales para aquellas empresas que se encuentran altamente restringidas en su capacidad. Específicamente, se concluye que las restricciones de capacidad son la principal fuente de la existencia de costes marginales crecientes.

Más recientemente, ha surgido una nueva rama de la literatura económica que analiza los efectos de las restricciones de capacidad en las dinámicas de exportación. El artículo de Ahn y McQuoid (2012) constituye un buen ejemplo de esta nueva literatura. En este trabajo, los autores desarrollan un modelo estructural sobre la participación en actividades de exportación que incorpora la existencia de restricciones de capacidad físicas y financieras. El modelo propuesto confirma la presencia de costes marginales crecientes en las empresas altamente restringidas, lo que limita la respuesta de estas empresas ante shocks externos de demanda. Adicionalmente, el artículo también señala que la existencia de un número elevado de empresas restringidas podría reducir significativamente el nivel de output agregado necesario para cubrir potenciales shocks de demanda externos, llevando a incrementar sustancialmente el nivel agregado de precios.

Resultados similares son obtenidos en el trabajo de Blum et al. (2013), quienes también enfatizan el papel de las restricciones de capacidad y de los shocks de demanda estocásticos en las decisiones de exportación de las empresas. A partir del nivel de inversión en capital fijo (variable utilizada como proxy para medir la existencia de restricciones de capacidad), el modelo propuesto permite predecir la forma de participación en las actividades de exportación: ocasional o permanente. Por un lado, los autores indican que los "exportadores ocasionales" son aquellos que deciden su potencial participación en la exportación en función del nivel de demanda doméstica y del grado de utilización de capital fijo. Por otro lado, definen los "exportadores permanentes" como aquellos que invierten una cantidad suficiente en capital fijo para abastecer ambos mercados, tanto el doméstico como el de exportación.

Otro ejemplo de esta nueva rama de la literatura que analiza la interconexión entre las dinámicas de exportación y las restricciones de capacidad es el trabajo de Soderbery (2014). Este artículo desarrolla un modelo de comercio internacional considerando la existencia de heterogeneidad tanto en los niveles de productividad como en el grado de utilización de la capacidad. El principal resultado del trabajo revela que el nivel de utilización de la capacidad está fuertemente determinado por el estatus exportador de la empresa y por el volumen total de ventas. Asimismo, el artículo también sugiere un potencial aumento en el precio de los bienes como consecuencia del incremento del número de empresas que se enfrentan a restricciones de capacidad. Dicho de otra forma, las empresas altamente restringidas únicamente pueden incrementar el precio de los bienes 
para aprovecharse de la entrada en nuevos mercados exteriores, dado que esa estrategia es el único mecanismo a su alcance para ajustar sus márgenes. ${ }^{4}$

Los trabajos anteriores son tres buenos exponentes de la reciente rama de la literatura que aborda los efectos de las restricciones de capacidad sobre las dinámicas de exportación. Para analizar el impacto de estas limitaciones en la realización de estrategias de $\mathrm{I}+\mathrm{D}$, es necesario partir de una premisa básica: las empresas suelen innovar, principalmente, para reducir costes (innovaciones de proceso) o para incrementar los niveles de demanda (innovaciones de producto). ${ }^{5}$ Por tanto, parece claro que variables relacionadas con el tamaño de la empresa, la utilización de la capacidad productiva, la estructura de mercado o las condiciones de la demanda podrían condicionar el desarrollo de esta actividad estratégica. En este sentido, el trabajo de Smolny (2003) podría ser un buen ejemplo de la literatura que ha abordado la interrelación entre las restricciones de capacidad y el desarrollo de actividades de I+D. Específicamente, el artículo propone un modelo de competencia monopolística que incorpora incertidumbre en los niveles de demanda y desequilibrios en el proceso de ajuste de utilización de la capacidad. La principal conclusión del trabajo sugiere que las expectativas de demanda a medio plazo y el grado de utilización de la capacidad productiva (variable proxy del nivel de demanda efectiva de la empresa) condicionan significativamente la implementación de innovaciones. Adicionalmente, también señala que la existencia de restricciones de capacidad reduce la probabilidad de llevar a cabo actividades de I+D.

Los factores que limitan la implementación de innovaciones son también analizados en Hewitt-Dundas (2006) y Oum et al. (2014). Por un lado, el trabajo de Hewitt-Dundas (2006) identifica tres tipos de restricciones esenciales que podrían condicionar la estrategia de I+D: financieras, organizativas y relativas al capital humano. Asimismo, también señala que son, fundamentalmente, las limitaciones relacionadas con las características específicas de las empresas las que influyen significativamente en el desarrollo de innovaciones y en el potencial éxito innovador. Por otro lado, el artículo de Oum et al. (2014) centra su atención en todas aquellas restricciones relacionadas con las características de las empresas que, de nuevo, dificultan la implantación de innovaciones. En este sentido, el trabajo indica que los principales determinantes que influyen en el desarrollo de esta actividad estratégica son la falta de información (vinculada, por ejemplo,

\footnotetext{
${ }^{4} \mathrm{Al}$ estar restringidas, las empresas no pueden incrementar libremente su nivel de producción para acceder a nuevos mercados exteriores. De esa forma, los únicos mecanismos disponibles para ajustar márgenes se derivarían de un incremento de precios o de la realización de costosas inversiones en capital fijo que aumenten el nivel de capacidad de la empresa.

${ }^{5}$ El principal objetivo de las innovaciones de proceso reside en la mejora de las técnicas de producción con el objetivo de aprovecharse de mayores economías de escala. Por el contrario, las innovaciones de producto buscan desarrollar productos diferenciados del resto de competidores y que estén mejor adaptados a las necesidades de la demanda.
} 
con las características del mercado o de los competidores), la escasez de capital y la mala cualificación del capital humano.

\section{DATOS Y RESULTADOS DESCRIPTIVOS}

El estudio que aquí se presenta se ha llevado a cabo utilizando información individualizada (microdatos) de empresas manufactureras españolas para el periodo 1990-2011. La base de datos que se ha usado es la Encuesta Sobre Estrategias Empresariales (ESEE), la cual es elaborada por la Fundación SEPI y el Ministerio de Industria, Turismo y Comercio. Esta base de datos utiliza el tamaño de la empresa y el sector de actividad CNAE (a dos dígitos) como principales criterios de estratificación. ${ }^{6}$ El número inicial de empresas para el periodo considerado asciende a 5.040.

A partir de esta muestra inicial, y con el fin de evitar la existencia de empresas que entran y salen de la encuesta, únicamente se han considerado aquellas empresas que cuentan con información disponible durante al menos tres años consecutivos. Adicionalmente, también se han eliminado las observaciones correspondientes al año 1990 debido a la falta de información en algunas variables relevantes. La muestra final, una vez aplicados los criterios anteriores, asciende a 36.700 observaciones correspondientes a 4.291 empresas.

La base de datos utilizada provee información detallada sobre las principales características de las empresas, entre las que se incluyen la participación en actividades de exportación e innovación. ${ }^{7}$ En este sentido, los datos muestran que el 35,05\% del total de empresas no ha exportado ni innovado a lo largo del periodo considerado, el 29,42\% únicamente ha exportado, el 4,47\% solamente ha participado en actividades de $\mathrm{I}+\mathrm{D}$, y, por último, el $31,06 \%$ ha realizado ambas actividades conjuntamente.

La ESEE también contiene información importante acerca de las condiciones de demanda del mercado. Por un lado, compara el estado de la demanda en un año específico en relación con el que existía en el periodo previo, distinguiendo entre tres escenarios fundamentales: recesión, estabilidad o expansión. ${ }^{8}$ Por otro lado, la base de datos utilizada también provee el grado de utilización de la

\footnotetext{
${ }^{6}$ La población de la ESEE únicamente considera las empresas manufactureras con 10 o más empleados. Por un lado, las empresas que emplean entre 10 y 200 trabajadores son seleccionadas aleatoriamente utilizando criterios basados en la clasificación CNAE. Por otro lado, todas las empresas que emplean a más de 200 trabajadores son invitadas a participar en la encuesta, obteniendo una tasa de participación cercana al 70\%.

${ }^{7}$ En relación a la participación en actividades de I+D, la ESEE permite distinguir entre innovaciones de proceso y de producto.

${ }^{8}$ La ESEE provee esta variable en cada uno de los cinco principales sectores de actividad donde la empresa opera. En este trabajo se ha considerado una media ponderada como indicador principal, donde los pesos de cada sector son la proporción de ventas sobre el total.
} 
capacidad productiva de las empresas (proxy del nivel de demanda). Esta variable toma valores en el intervalo [0 - 100], donde valores altos cercanos a 100 podrían sugerir la existencia de restricciones de capacidad productiva. El grado medio de utilización de la capacidad para el periodo considerado es aproximadamente el 80\%, con una desviación típica del 16\%.

Los dos gráficos siguientes presentan un análisis descriptivo básico sobre la variable que mide el nivel de utilización de la capacidad productiva de las empresas. Así, la Figura 1(a) muestra la distribución de esta variable para la muestra de empresas manufactureras españolas. Como puede verse en este gráfico, alrededor del 50\% (25\%) de las empresas de la muestra presenta una tasa de utilización superior al $80 \%$ (90\%). La figura también indica que aproximadamente el $17 \%$ del total de empresas utilizan su capacidad productiva a pleno rendimiento, esto es, su nivel de utilización de la capacidad es igual al 100\%. Adicionalmente, la Figura 1(b) representa la evolución del nivel medio de utilización a lo largo del periodo considerado. Como era de esperar, el gráfico revela el fuerte comportamiento procíclico de esta variable. En concreto, la figura muestra una caída significativa de los niveles medios de utilización de la capacidad durante los periodos de crisis. Esta caída ha sido especialmente significativa durante el reciente periodo de crisis económica y financiera mundial, donde este indicador pasó del 83,1\% en 2007 al 72,1\% en $2011 .{ }^{9}$

Figura 1

Utilización de la capacidad productiva (1990-2011)

a) Histograma de la utilización de capacidad

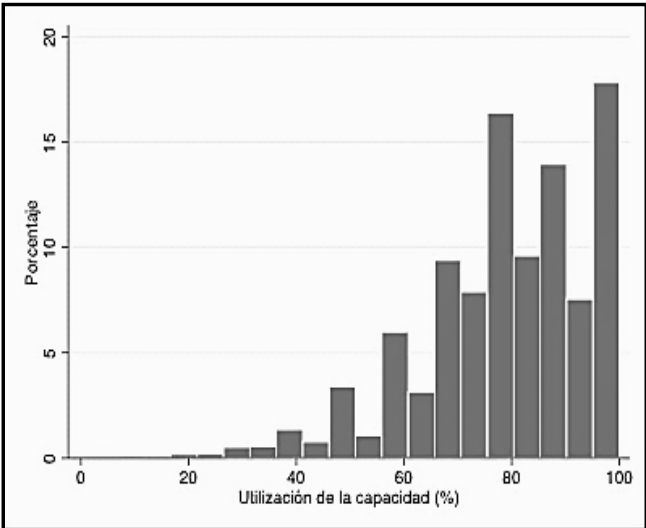

b) Evolución de la utilización media

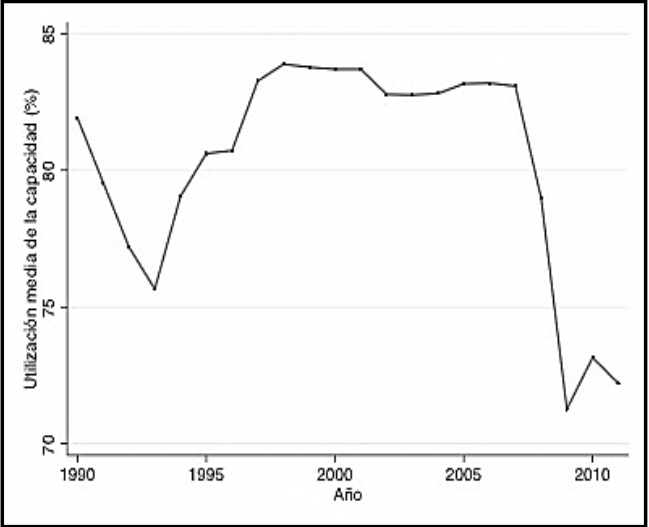

Fuente: Elaboración propia a partir de los datos de la ESEE.

\footnotetext{
${ }^{9}$ El grado medio de utilización de la capacidad productiva de las empresas es también calculado por el Ministerio de Industria, Turismo y Comercio. La evolución de este indicador es muy similar a la que se muestra en la Figura 1(b), destacando de nuevo la fuerte caída del periodo 2007-2011.
} 
Para analizar la relación existente entre la utilización de la capacidad de la empresa y la participación en actividades de exportación e innovación, la Tabla 1 muestra el porcentaje de empresas que llevan a cabo estas actividades agrupadas en función de su nivel de utilización de la capacidad. Como se puede observar, parece claro que existe una correlación positiva entre el grado de utilización de la capacidad y la participación en estas actividades estratégicas. Por un lado, los datos muestran que tener una alta tasa de utilización incrementa la probabilidad de exportar. Específicamente, los datos sugieren que la exportación es más probable en las empresas cuyo grado de utilización es superior al $40 \%$ (i.e., $55,45 \%$ vs. $44,55 \%$ para el grupo $41-60$; ó $65,25 \%$ vs. 34,75\% para el grupo 81 100). Por otro lado, la Tabla 1 también revela que las empresas son menos propensas a innovar que a exportar. Sin embargo, y al igual que sucedía con la exportación, tener una mayor utilización de la capacidad productiva incrementa la probabilidad de llevar a cabo actividades de I+D.

Tabla 1

Utilización de la capacidad productiva, exportación e I+D (\% de empresas)

\begin{tabular}{rcc|cc|c}
\hline $\begin{array}{c}\text { Nivel de } \\
\text { utilización }\end{array}$ & \multicolumn{2}{c|}{ Exportación } & \multicolumn{2}{c|}{ I+D } & $\begin{array}{c}\% \text { de } \\
\text { empresas }\end{array}$ \\
\hline $0-20$ & 56,30 & So & No & Sí & 0,37 \\
$21-40$ & 56,95 & 43,70 & 82,71 & 17,29 & 2,65 \\
$41-60$ & 44,55 & 55,45 & 79,92 & 20,08 & 11,31 \\
$61-80$ & 38,41 & 61,59 & 60,64 & 29,36 & 36,78 \\
$81-100$ & 34,75 & 65,25 & 61,55 & 35,32 & 48,89 \\
\hline
\end{tabular}

Fuente: Elaboración propia a partir de los datos de la ESEE.

Como se mencionó en la introducción del artículo, el principal objetivo del trabajo es evaluar el impacto de las restricciones de capacidad productiva sobre la decisión conjunta de exportar e innovar. Para llevar a cabo este análisis es necesario, por tanto, definir los principales criterios que podrían sugerir la presencia de estas restricciones. En primer lugar, y siguiendo los criterios propuestos por Smolny (2003) y Ahn y McQuoid (2012), se han utilizado dos umbrales distintos relacionados con el nivel de utilización de la capacidad productiva que indicarían que la empresa se encuentra restringida. Así, se va a considerar que una empresa se enfrenta a restricciones de capacidad cuando su nivel de utilización de la capacidad es superior al 95\% ( $U>95)$ o igual al 100\% $(U=100) .{ }^{10}$ En segundo lugar, y siguiendo el criterio propuesto en Crespo y Muñoz-Sepúlveda (2015) que permite incorporar heterogeneidad a nivel industria

${ }^{10}$ Smolny (2003) considera que una empresa está restringida cuando su nivel de utilización de la capacidad es superior al 95\%, mientras que Ahn y McQuoid (2012) asumen que este umbral debe ser igual al $100 \%$. 
y tiempo, se ha calculado un umbral específico para cada sector $j$ y año $t .^{11}$ Específicamente, el nuevo umbral $\left(U>U_{j t}^{*}\right.$ ) se define como la suma de (i) la tasa media de utilización de la capacidad para cada industria $j$ y año $t$, y (ii) la desviación típica de esta variable, de nuevo, para cada industria y año. En este sentido, la inclusión de la desviación estándar en el cálculo del umbral permite controlar por la dispersión media del sector a lo largo del tiempo. De esta forma, la empresa perteneciente a la industria $j$ se enfrentará a restricciones de capacidad en el año $t$ si su tasa de utilización de la capacidad es superior al umbral calculado para dicha industria $j$ en el mismo año específico $t$.

Tabla 2

Restricciones de capacidad productiva, exportación e I+D (\% de empresas)

\begin{tabular}{lcc|cc|cc|c}
\hline & \multicolumn{2}{c|}{ Exportación } & \multicolumn{2}{c|}{ I+D } & \multicolumn{2}{c|}{ Exportación e I+D } & \multicolumn{2}{c}{ \% de } \\
& No & Sí & No & Sí & No & Sí & empresas \\
\hline$U>95$ (restringida) & 7,09 & 10,22 & 11,87 & 5,45 & 12,46 & 4,84 & 17,33 \\
$U \leq 95$ (no restringida) & 30,78 & 51,91 & 52,42 & 30,25 & 56,01 & 26,69 & 82,67 \\
\hline$U=100$ (restringida) & 6,63 & 7,88 & 10,60 & 3,92 & 11,13 & 3,38 & 14,52 \\
$U<100$ (no restringida) & 31,24 & 54,24 & 53,70 & 31,78 & 57,35 & 28,14 & 85,48 \\
\hline$U>U_{j t}^{*}$ (restringida) & 7,30 & 10,22 & 12,09 & 5,44 & 12,70 & 4,80 & 17,54 \\
$U \leq U_{j t}^{*}$ (no restringida) & 30,57 & 51,91 & 52,21 & 30,26 & 55,77 & 26,72 & 82,46 \\
\hline
\end{tabular}

Fuente: Elaboración propia a partir de los datos de la ESEE.

La Tabla 2 muestra el porcentaje de empresas que participan en actividades de exportación e I+D (o en ambas actividades de forma conjunta) distinguiendo entre empresas restringidas y no restringidas en función de los tres criterios o umbrales considerados $\left(U>95, U=100 \mathrm{y} U>U_{j t}^{*}\right)$. Como puede observarse, parece evidente que la existencia de restricciones de capacidad condiciona la potencial participación en estas actividades estratégicas. En primer lugar, los resultados revelan que, considerando el umbral de utilización de la capacidad del $95 \%$, aproximadamente el $17,3 \%$ de las empresas de la muestra se enfrentan a restricciones de capacidad. Este porcentaje es muy similar al obtenido cuando se tiene en cuenta el umbral que incorpora heterogeneidad a nivel industria y tiempo, y ligeramente superior al obtenido cuando dicho se umbral se establece en el $100 \%$.

En segundo lugar, los datos muestran una mayor probabilidad de exportar en

${ }^{11} \mathrm{El}$ principal supuesto que asume este trabajo sugiere que el umbral que determina la existencia de restricciones de capacidad varía entre sectores y a lo largo del tiempo. Ver Crespo y MuñozSepúlveda (2015) para más detalles. 
aquellas empresas que no se enfrentan a limitaciones en su capacidad productiva. Específicamente, los resultados indican que la exportación es un 69\% más probable en las empresas sin restricciones (51,91 vs. 30,78 utilizando el umbral de utilización del 95\%). Asimismo, y considerando únicamente a las empresas restringidas, los datos también revelan que la exportación es un $44 \%$ más probable en este último tipo de empresas (10,22 vs. 7,09). Respecto a la estrategia de innovación, los resultados muestran, por el contrario, una menor propensión a innovar en las empresas. Sin embargo, y al igual que sucedía con la exportación, la no presencia de limitaciones de capacidad favorece el desarrollo de este tipo de actividades. En concreto, mientras que solamente el $31,4 \%$ de las empresas restringidas lleva a cabo actividades de I+D, este porcentaje asciende al $36,6 \%$ cuando se considera a las empresas no limitadas en su capacidad.

En resumen, los resultados descriptivos anteriores podrían confirmar la existencia de un mejor proceso de ajuste de los niveles de utilización de la capacidad en aquellas empresas que no se enfrentan a restricciones productivas. Este mejor ajuste podría desempeñar, sin duda, un papel relevante en el acceso a nuevos mercados exteriores o en la mayor realización de actividades de I+D.

\section{ANÁLISIS ECONOMÉTRICO}

La presente sección va a aplicar un modelo Probit bivariado para evaluar econométricamente el impacto de las restricciones de capacidad productiva (y de otro conjunto de variables relevantes) sobre la decisión conjunta de exportar e innovar. ${ }^{12}$ Específicamente, el modelo Probit bivariado que se va a estimar es el siguiente:

$$
\begin{aligned}
& X_{i t}=\left\{\begin{array}{rrr}
1, & \text { si } & \beta^{X} C_{i t-1}+\gamma^{X} W_{i t-1}+\eta^{X} y_{t}+\lambda^{X} s_{i}+\varepsilon_{i t}^{X}>0 \\
0, & \text { en caso contrario }
\end{array}\right. \\
& I+D_{i t}=\left\{\begin{array}{lcc}
1, & \text { si } & \beta^{I+D} C_{i t-1}+\gamma^{I+D} W_{i t-1}+\eta^{I+D} y_{t}+\lambda^{I+D} s_{i}+\varepsilon_{i t}^{I+D}>0 \\
0, & \text { en caso contrario }
\end{array}\right.
\end{aligned}
$$

Como puede observarse en las ecuaciones (1) y (2), la decisión de exportar $(X)$ y de innovar $(I+D)$ de la empresa $i$ en el periodo $t$ se hace depender de un conjunto de variables $C$ relacionadas con la capacidad productiva de la empresa en el periodo previo, y de otro conjunto de variables retardadas $W$. Adicionalmente, el análisis incluye dos grupos de variables dummies que

${ }^{12}$ A diferencia de los trabajos previos sobre este tema que analizan la decisión exportadora e innovadora de la empresa desde una perspectiva continua (véase, entre otros, Ahn y McQuoid, 2012; Soderbery, 2014; o Crespo y Muñoz-Sepúlveda, 2015), este trabajo únicamente evalúa decisiones binarias sobre la potencial participación en actividades de exportación e I+D (exporta/no exporta e innova/no innova). 
permiten considerar la existencia de efectos temporales $(y)$ y sectoriales (s), definidos estos últimos a dos dígitos CNAE. El modelo es estimado utilizando métodos de máxima verosimilitud, considerando el supuesto de que los errores de las ecuaciones (1) y (2) están correlacionados.

Siguiendo la metodología propuesta por Smolny (2003) y Ahn y McQuoid (2012), las variables que se recogen en el vector $C_{i t-1}$ son dos. Por un lado, este vector incluye el nivel estándar de utilización de la capacidad productiva de la empresa $(U)$, el cual se espera que influya positivamente, hasta un determinado umbral, sobre la probabilidad de llevar a cabo ambas actividades estratégicas. Por otro lado, se incluye también una variable dummy vinculada con la posible existencia de restricciones de capacidad productiva. Específicamente, esta variable toma el valor 1 cuando el nivel de utilización de la capacidad de la empresa es superior a un umbral determinado. En este sentido, y como se explicó anteriormente, se van a considerar tres umbrales diferentes que podrían sugerir la existencia de limitaciones productivas: $U>95, U=100$ y $U>U_{j t}^{*}$. Dado que las empresas fuertemente restringidas se enfrentan con importantes limitaciones productivas, se espera que esta variable tenga un efecto negativo sobre la probabilidad de exportar y realizar I+D.

Adicionalmente, el vector $W_{i t-1}$ recoge un conjunto de variables relevantes a nivel de empresa que podrían influir significativamente en el desarrollo de estas actividades estratégicas. En primer lugar, este vector incluye tres variables dummies relacionadas con el tamaño de la empresa. Así, se diferencia entre empresas Pequeñas, Medianas y Grandes, según estas empleen menos de 50, entre 50 y 200, y más de 200 trabajadores, respectivamente. En segundo lugar, se ha estimado una medida de la Productividad Total de los Factores (PTF) utilizando el enfoque de Levinsohn y Petrin (2003). ${ }^{13}$ En tercer lugar, este vector recoge también una medida de la estructura de propiedad de la empresa. Específicamente, la variable Propiedad toma valor 1 si la empresa está controlada principalmente por capital extranjero, y cero en caso contrario. Por último, el vector $W_{i t-1}$ incluye dos variables dummies vinculadas con el estado actual de la demanda. De esta forma, la variable Recesivo (Expansivo) toma valor uno cuando el estado es claramente recesivo (expansivo) respecto al existente en el periodo previo, y cero en caso contrario.

La Tabla 3 muestra los resultados de la estimación del modelo Probit bivariado sobre la decisión conjunta de exportar e innovar, considerando los tres umbrales diferentes que podrían determinar la existencia de restricciones de

${ }^{13}$ Levinsohn y Petrin (2003) proponen un modelo semiparamétrico que utiliza los consumos intermedios (e.g. materias primas o energía) como proxy de los niveles de productividad no observados. 
capacidad. Como puede observarse, el parámetro rho estimado -medida de la correlación entre los errores de las ecuaciones (1) y (2)- es, en todos los casos, positivo y significativamente distinto de cero. Este resultado indica que la estimación simultánea del modelo genera resultados más eficientes que los obtenidos en el proceso de estimación univariante (Greene, 2011).

Tabla 3

Resultados del modelo Probit bivariado sobre la decisión de exportar e innovar

\begin{tabular}{|c|c|c|c|c|c|c|}
\hline & \multicolumn{2}{|c|}{$U>95$} & \multicolumn{2}{|c|}{$U=100$} & \multicolumn{2}{|c|}{$U>U_{j t}^{*}$} \\
\hline & $x$ & $I+D$ & $x$ & $I+D$ & $x$ & $I+D$ \\
\hline$U$ & $\begin{array}{l}0,135^{\star \star} \\
(0,068)\end{array}$ & $\begin{array}{l}0,144^{\star *} \\
(0,071)\end{array}$ & $\begin{array}{c}0,219^{* \star *} \\
(0,066)\end{array}$ & $\begin{array}{c}0,199^{* \star *} \\
(0,069)\end{array}$ & $\begin{array}{c}0,188^{\star * \star} \\
(0,068)\end{array}$ & $\begin{array}{l}0,162^{\star *} \\
(0,071)\end{array}$ \\
\hline$U>95$ & $\begin{array}{c}-0,080^{* * *} \\
(0,027)\end{array}$ & $\begin{array}{c}-0,145^{\star \star *} \\
(0,027)\end{array}$ & - & - & - & - \\
\hline$U=100$ & - & - & $\begin{array}{c}-0,156^{\star \star \star} \\
(0,028)\end{array}$ & $\begin{array}{c}-0,218^{\star \star \star} \\
(0,029)\end{array}$ & - & - \\
\hline$U>U_{j t}^{*}$ & - & - & - & - & $\begin{array}{c}-0,114^{\star \star *} \\
(0,027)\end{array}$ & $\begin{array}{c}-0,154^{\star \star *} \\
(0,027)\end{array}$ \\
\hline Pequeñas & $\begin{array}{c}-0,607^{\star \star \star} \\
(0,025)\end{array}$ & $\begin{array}{c}-0,597^{\star \star \star} \\
(0,025)\end{array}$ & $\begin{array}{c}-0,604^{\star \star \star} \\
(0,025)\end{array}$ & $\begin{array}{c}-0,593^{\star \star \star} \\
(0,025)\end{array}$ & $\begin{array}{c}-0,606^{\star \star *} \\
(0,025)\end{array}$ & $\begin{array}{c}-0,596^{\star \star \star} \\
(0,025)\end{array}$ \\
\hline Grandes & $\begin{array}{c}0,395^{\star \star \star} \\
(0,029)\end{array}$ & $\begin{array}{c}0,587^{\star \star \star} \\
(0,025)\end{array}$ & $\begin{array}{c}0,392^{\star \star \star} \\
(0,029)\end{array}$ & $\begin{array}{c}0,585^{\star \star \star} \\
(0,025)\end{array}$ & $\begin{array}{c}0,393^{\star \star \star} \\
(0,029)\end{array}$ & $\begin{array}{c}0,586^{\star \star \star} \\
(0,025)\end{array}$ \\
\hline PTF & $\begin{array}{c}0,673^{\text {*** }}(0,027)\end{array}$ & $\begin{array}{c}0,531^{* \star *} \\
(0,026)\end{array}$ & $\begin{array}{c}0,669 * * * \\
(0,027)\end{array}$ & $\begin{array}{c}0,527^{\star * \star *} \\
(0,026)\end{array}$ & $\begin{array}{c}0,672^{* * *} \\
(0,027)\end{array}$ & $\begin{array}{c}0,531^{\star \star *} \\
(0,026)\end{array}$ \\
\hline Propiedad & $\begin{array}{c}0,398^{\star * *} \\
(0,031)\end{array}$ & $\begin{array}{l}-0,041^{*} \\
(0,024)\end{array}$ & $\begin{array}{c}0,399^{* * *} \\
(0,031)\end{array}$ & $\begin{array}{l}-0,041^{*} \\
(0,024)\end{array}$ & $\begin{array}{c}0,399 * * * \\
(0,031)\end{array}$ & $\begin{array}{l}-0,041^{*} \\
(0,024)\end{array}$ \\
\hline Recesivo & $\begin{array}{c}0,152^{\star \star * *} \\
(0,021)\end{array}$ & $\begin{array}{c}0,123^{\star * * *} \\
(0,022)\end{array}$ & $\begin{array}{c}0,152^{\star * *} \\
(0,021)\end{array}$ & $\begin{array}{c}0,123^{\star * *} \\
(0,022)\end{array}$ & $\begin{array}{c}0,152^{\star \star *} \\
(0,021)\end{array}$ & $\begin{array}{c}0,123^{\star * *} \\
(0,022)\end{array}$ \\
\hline Expansivo & $\begin{array}{c}0,174^{\star \star *} \\
(0,021)\end{array}$ & $\begin{array}{c}0,249 * \star * \\
(0,021)\end{array}$ & $\begin{array}{c}0,173^{\star \star * *} \\
(0,021)\end{array}$ & $\begin{array}{c}0,248^{\star \star * *} \\
(0,021)\end{array}$ & $\begin{array}{c}0,173^{\star * *} \\
(0,021)\end{array}$ & $\begin{array}{c}0,249 \text { *** } \\
(0,021)\end{array}$ \\
\hline $\begin{array}{l}\text { Observaciones } \\
\text { Log-Likelihood } \\
\text { Rho }(\rho) \\
\text { Test } \rho=0\end{array}$ & $\begin{array}{r}-28 \\
0,35 \\
x^{2}(1 \\
p-v e\end{array}$ & $\begin{array}{l} \\
64 \\
013) \\
4,65 \\
0,0\end{array}$ & $\begin{array}{r}3 \\
-28 \\
0,35 \\
x^{2}(1 \\
p-v\end{array}$ & $\begin{array}{l}9 \\
95 \\
013) \\
7,68 \\
0,0\end{array}$ & $\begin{array}{r}3 \\
-28 \\
0,35 \\
x^{2}(1 \\
p-v a\end{array}$ & $\begin{array}{l}46 \\
4613) \\
3,32 \\
0,0\end{array}$ \\
\hline
\end{tabular}

Nota: Todas las variables explicativas están retardadas un periodo. ***, **, * indica significativo al 1\%, al 5\% y al 10\%, respectivamente. Errores estándar (robustos) de los estimadores entre paréntesis. Todas las estimaciones incluyen dummies temporales y sectoriales.

Fuente: Estimación propia a partir de los datos de la ESEE.

Los resultados vinculados con la decisión de exportar confirman lo obtenido en el análisis descriptivo de los datos. Por un lado, se obtiene una relación positiva entre la tasa de utilización de la capacidad y la probabilidad de exportar. Este resultado indica que las empresas con mayores tasas de utilización de la capacidad en el periodo previo son más propensas a exportar. Por otro lado, la Tabla 3 también confirma el impacto negativo de las restricciones de capacidad 
productiva sobre la decisión exportadora de la empresa. ${ }^{14}$ Dicho de otra forma, la existencia de restricciones de capacidad limita la respuesta de las empresas restringidas ante shocks externos de demanda, reduciendo la probabilidad de llevar a cabo actividades de exportación. El resto de variables de control consideradas en el análisis muestran el signo esperado. Así, por ejemplo, los resultados revelan una influencia positiva y significativa del tamaño de la empresa y del nivel de productividad sobre la probabilidad de exportar.

Respecto a la probabilidad de llevar a cabo actividades de I+D, los resultados son similares a los mencionados anteriormente. En primer lugar, la Tabla 3 también confirma el impacto positivo de la tasa de utilización de la capacidad sobre la probabilidad de innovar. Al igual que sucedía con la exportación, este resultado sugiere que las empresas son más propensas a realizar I+D cuando sus niveles de utilización de la capacidad en el periodo previo son altos (pero sin llegar a enfrentarse a restricciones de capacidad). Así, por ejemplo, uno de los objetivos de este proceso de innovación podría estar relacionado con la mejora del proceso productivo (innovaciones de proceso), con el fin de incrementar la capacidad productiva de la empresa y evitar la aparición de restricciones de capacidad. En segundo lugar, los resultados también muestran el impacto negativo de las limitaciones de capacidad sobre la probabilidad de innovar. Este resultado indica que las empresas restringidas que cuentan con altas tasas de utilización de la capacidad y que se enfrentan, a corto plazo, a un lento proceso de ajuste entre los factores productivos y los niveles observados de demanda no pueden modificar fácilmente las decisiones previas tomadas a largo plazo sobre la potencial implementación de innovaciones. ${ }^{15}$ Por último, el resto de variables relevantes a nivel empresa tienen el signo esperado, destacando de nuevo la relación positiva entre el tamaño de la empresa y el nivel de productividad sobre la probabilidad de llevar a cabo actividades de I+D.

\subsection{Innovaciones de producto y de proceso}

Los resultados presentados en la sección anterior han puesto de manifiesto el papel relevante de las restricciones de capacidad productiva en la decisión conjunta de exportar e innovar. En este sentido, sin embargo, la variable dummy relacionada con la participación en actividades de I+D no establecía ninguna distinción entre el tipo de innovación llevada a cabo por la empresa (únicamente consideraba si la empresa había dedicado algún recurso a alguna actividad de

${ }^{14}$ Este resultado es obtenido considerando los tres umbrales distintos de utilización de la capacidad que podrían sugerir la existencia de restricciones productivas.

${ }^{15}$ Smolny (2003) asume que las empresas determinan su nivel de utilización de la capacidad y su potencial participación en actividades de I+D a largo plazo, y considerando la presencia de elevados niveles de incertidumbre sobre la demanda futura. Este supuesto podría dificultar, por tanto, la implementación de nuevas innovaciones ante desequilibrios de la empresa a corto plazo. 
I+D). Para abordar este aspecto, esta sección va a analizar de nuevo el efecto de las limitaciones de capacidad sobre la probabilidad conjunta de exportar e innovar, diferenciando en este último caso entre innovaciones de producto e innovaciones de proceso.

Como se mencionó anteriormente, la base de datos utilizada permite diferenciar entre estos dos tipos principales de innovación. Más específicamente, la ESEE contiene dos variables dummies relacionadas con la participación en actividades de innovación de producto y de proceso. En este contexto, se entiende por innovación de producto como el conjunto de actividades de I+D orientadas a implementar o comercializar nuevos productos con características mejoradas. Por el contrario, las innovaciones de proceso recogerían todas aquellas actividades de innovación enfocadas a mejorar las técnicas de producción de la empresa.

Tabla 4

Resultados del modelo Probit bivariado sobre la decisión de exportar e innovar en producto y en proceso

\begin{tabular}{|c|c|c|c|c|}
\hline & \multicolumn{2}{|c|}{ Innovación de producto } & \multicolumn{2}{|c|}{ Innovación de proceso } \\
\hline & $x$ & $I+D$ & $x$ & $I+D$ \\
\hline$U$ & $\begin{array}{l}0,120^{\star \star \star} \\
(0,041)\end{array}$ & $\begin{array}{c}0,034 \\
(0,043)\end{array}$ & $\begin{array}{c}0,189^{\star \star \star} \\
(0,068)\end{array}$ & $\begin{array}{c}0,358^{\star \star \star *} \\
(0,065)\end{array}$ \\
\hline$U>U_{j t}^{*}$ & $\begin{array}{c}-0,110^{\star * *} \\
(0,025)\end{array}$ & $\begin{array}{c}-0,121^{* * *} \\
(0,025)\end{array}$ & $\begin{array}{c}-0,117^{\star * *} \\
(0,027)\end{array}$ & $\begin{array}{c}-0,179 * * * \\
(0,025)\end{array}$ \\
\hline Pequeñas & $\begin{array}{c}-0,603^{\star \star *} \\
(0,025)\end{array}$ & $\begin{array}{c}-0,314^{\star \star *} \\
(0,025)\end{array}$ & $\begin{array}{c}-0,601^{\star \star *} \\
(0,025)\end{array}$ & $\begin{array}{c}-0,200^{\star \star *} \\
(0,024)\end{array}$ \\
\hline Grandes & $\begin{array}{c}0,388^{\star * \star} \\
(0,029)\end{array}$ & $\begin{array}{c}0,274^{\star \star \star} \\
(0,025)\end{array}$ & $\begin{array}{c}0,391 * \star \star \\
(0,030)\end{array}$ & $\begin{array}{c}0,260 * * * \\
(0,024)\end{array}$ \\
\hline PTF & $\begin{array}{c}0,677^{\star \star *} \\
(0,028)\end{array}$ & $\begin{array}{l}0,180^{\star \star *} \\
(0,024)\end{array}$ & $\begin{array}{c}0,678^{\star \star *} \\
(0,028)\end{array}$ & $\begin{array}{c}0,271^{\star \star *} \\
(0,023)\end{array}$ \\
\hline Propiedad & $\begin{array}{l}0,403^{\star \star \star} \\
(0,031)\end{array}$ & $\begin{array}{l}-0,058^{\star \star} \\
(0,023)\end{array}$ & $\begin{array}{c}0,411^{\star \star \star} \\
(0,031)\end{array}$ & $\begin{array}{l}-0,035 \\
(0,022)\end{array}$ \\
\hline Recesivo & $\begin{array}{c}0,154^{\star \star *} \\
(0,021)\end{array}$ & $\begin{array}{l}0,103^{\star \star \star} \\
(0,022)\end{array}$ & $\begin{array}{c}0,154^{\star \star *} \\
(0,021)\end{array}$ & $\begin{array}{c}0,018 \\
(0,020)\end{array}$ \\
\hline Expansivo & $\begin{array}{c}0,171^{\star * *} \\
(0,021)\end{array}$ & $\begin{array}{c}0,250 * \star * \\
(0,020)\end{array}$ & $\begin{array}{c}0,170^{\star * *} \\
(0,021)\end{array}$ & $\begin{array}{c}0,299 * * * \\
(0,019)\end{array}$ \\
\hline Observaciones & \multicolumn{2}{|c|}{30774} & \multicolumn{2}{|c|}{30774} \\
\hline Log-Likelihood & \multicolumn{2}{|c|}{$-29463,40$} & \multicolumn{2}{|c|}{$-29455,95$} \\
\hline Rho $(\rho)$ & \multicolumn{2}{|c|}{$0,278(0,013)$} & \multicolumn{2}{|c|}{$0,278(0,013)$} \\
\hline Test $\rho=0$ & \multicolumn{2}{|c|}{$\begin{array}{c}x^{2}(1)=446,52 \\
p \text {-value }=0,0\end{array}$} & \multicolumn{2}{|c|}{$\begin{array}{c}x^{2}(1)=444,98 \\
p \text {-value }=0,0\end{array}$} \\
\hline
\end{tabular}

Nota: Todas las variables explicativas están retardadas un periodo. ***, **, * indica significativo al $1 \%$, al $5 \%$ y al 10\%, respectivamente. Errores estándar (robustos) de los estimadores entre paréntesis. Todas las estimaciones incluyen dummies temporales y sectoriales.

Fuente: Estimación propia a partir de los datos de la ESEE.

La Tabla 4 muestra los resultados del modelo Probit bivariado sobre la decisión de exportar e innovar, distinguiendo en este último caso entre 
innovaciones de producto y de proceso. ${ }^{16}$ Como se observa, el parámetro rho que mide la correlación entre errores es positivo y significativo, lo que indica que ambas ecuaciones necesitan ser estimadas simultáneamente. Los resultados también confirman el impacto negativo de las restricciones de capacidad sobre la probabilidad de exportar e innovar en producto y en proceso. Dicho de otra forma, las empresas que se enfrentan a restricciones de capacidad son menos propensas a llevar a cabo actividades de I+D, tanto de producto como de proceso. Adicionalmente, la Tabla 4 muestra un resultado interesante relacionado con el efecto de la tasa de utilización de la capacidad. Por un lado, se observa que el nivel previo de utilización de la capacidad no influye en la probabilidad de llevar a cabo innovaciones de producto. Como es sabido, la implantación de este tipo de innovaciones no se basa en el nivel de utilización de la capacidad de la empresa sino que únicamente se centra en la mejora de las características del producto para conseguir una mejor adecuación a las necesidades de la demanda. Por otro lado, la Tabla 4 sí que revela un impacto positivo de la tasa de utilización de la capacidad en el periodo previo sobre la probabilidad de realizar innovaciones de proceso. En general, estas innovaciones buscan mejorar el proceso productivo de la empresa, de manera que aquellas empresas con altos niveles de utilización de la capacidad (pero sin llegar a estar restringidas) podrían optar por desarrollar este tipo de innovaciones con el objetivo de mejorar su capacidad productiva. Finalmente, el resto de variables consideradas en el análisis muestran el efecto esperado.

\section{CONCLUSIONES}

La reciente crisis económica y financiera mundial que comenzó a finales de 2007 ha puesto de manifiesto el importante papel que desempeñan las restricciones de capacidad (físicas y financieras) en las principales decisiones estratégicas de las empresas. Así, por ejemplo, en los últimos años ha surgido una nueva rama de la literatura económica que se ha centrado en evaluar el impacto de estas restricciones en las dinámicas de exportación e innovación. En este contexto, este artículo pretende evaluar los principales determinantes que influyen en la decisión conjunta de exportar e innovar, prestando una especial atención en el impacto de las restricciones físicas de capacidad. La principal hipótesis que se pretende contrastar es si las empresas fuertemente restringidas son menos propensas a participar en actividades de exportación e inversión en $\mathrm{I}+\mathrm{D}$.

${ }^{16}$ La Tabla 4 únicamente considera como medida de la existencia de restricciones de capacidad el umbral que incorpora heterogeneidad temporal y sectorial $\left(U>U_{j t}^{*}\right.$ ). Los resultados obtenidos al considerar el resto de umbrales $(U>95$ y $U=100)$ son similares a los que se muestran en la tabla. 
El análisis empírico se ha llevado a cabo utilizando los datos de una muestra representativa de empresas manufactureras españolas (la Encuesta Sobre Estrategias Empresariales, ESEE) para el periodo 1990-2011. A partir de estos datos, el artículo estima un modelo Probit bivariado para evaluar el impacto de las restricciones físicas de capacidad (y de otras variables relevantes a nivel empresa) sobre la probabilidad conjunta de exportar y realizar I+D. El análisis realizado confirma la importancia del nivel previo de utilización de la capacidad productiva de la empresa en la potencial participación en estas actividades. Así, los resultados obtenidos sugieren una mayor probabilidad de exportar e invertir en $\mathrm{I}+\mathrm{D}$ cuanto mayor sea el nivel de utilización de la capacidad de la empresa en el periodo anterior. En este sentido, sin embargo, los resultados también indican que superar un determinado nivel o umbral muy elevado de utilización de la capacidad (proxy de la existencia de restricciones de capacidad) reduce significativamente la probabilidad de participar en estas estrategias. Este resultado confirmaría, por tanto, el papel negativo que desempeñan las restricciones físicas de capacidad en la decisión conjunta de exportar e invertir en I+D. Por último, y como era de esperar, el análisis econométrico destaca la relación positiva y significativa del tamaño y del nivel de productividad de la empresa sobre la probabilidad de realizar ambas actividades.

Adicionalmente, el artículo también estima un modelo Probit bivariado para evaluar de nuevo el impacto de las limitaciones de capacidad sobre la probabilidad conjunta de exportar e innovar, distinguiendo en este último caso entre innovaciones de producto e innovaciones de proceso. El análisis realizado enfatiza de nuevo el impacto negativo de las restricciones de capacidad sobre la probabilidad de llevar a cabo ambas actividades de I+D.

Los resultados presentados en este trabajo podrían sugerir el desarrollo de políticas económicas orientadas a mejorar la capacidad productiva de las empresas, con el fin de lograr un mejor ajuste entre los niveles potenciales de demanda y los inputs fijos necesarios en el proceso productivo. Evidentemente, esta mejora del proceso de ajuste dotaría a las empresas de un mayor de grado de flexibilidad y adaptación frente a shocks externos de demanda, lo que podría incentivar la entrada en nuevos mercados exteriores o el desarrollo de mayores inversiones en $\mathrm{I}+\mathrm{D}$.

Finalmente, este trabajo permite identificar otras líneas de investigación futuras. Por un lado, sería interesante incluir también en el análisis el impacto de las restricciones financieras. Como se ha mencionado en la sección de literatura previa, las restricciones de capacidad (tanto físicas como financieras) modifican sustancialmente la estructura de costes marginales de la empresa, siendo la principal fuente de la existencia de costes marginales crecientes. El presente trabajo ha confirmado el impacto negativo de las restricciones físicas de capacidad sobre la decisión conjunta de exportar e innovar, pero no ha 
considerado el papel de las restricciones financieras. Así, una futura línea de investigación estaría relacionada con la inclusión de variables que permitan identificar la presencia y el impacto de estas restricciones. Por otro lado, sería necesario considerar también el carácter dinámico de las decisiones estratégicas de exportar e invertir en I+D. En este sentido, una futura línea de investigación debería incluir también decisiones previas tomadas por la empresa en periodos anteriores a $t-1$, de forma que se pueda evaluar con una mayor profundidad el carácter dinámico de ambas estrategias.

\section{REFERENCIAS BIBLIOGRÁFICAS}

AHN, J. y MCQUOID, A.F. (2012). "Capacity Constrained Exporters: Micro Evidence and Macro Implications". Economic Research Working Paper Series 11-2012, Florida International University. http://digitalcommons.fiu.edu/cgi/viewcontent.cgi?article= 1001\&context=economics_wps. [Último acceso: Septiembre de 2016].

BERNARD, A.B.; EATON, J.; JENSEN, J.B. y KORTUM, S. (2003). "Plants and Productivity in International Trade". American Economic Review, 93(4), pp. 1268-1290.

BLUM, B.S.; CLARO, S. y HORSTMANN, I.J. (2013). "Occasional and perennial exporters". Journal of International Economics, 90, pp. 64-75.

CRESPO, A. y MUÑOZ-SEPÚLVEDA, J.A. (2015). "The role of physical and financial constraints in export dynamics". Economics Working Papers MWP 2015/17, European University Institute.

GREEN, W.H. (2011). "Econometric Analysis" (7th International Edition). Pearson Education.

HELPMAN, E.; MELITZ, M.J. y YEAPLE, S.R. (2004). "Export Versus FDI with Heterogeneous Firms". American Economic Review, 94(1), pp. 300-316.

HEWITT-DUNDAS, N. (2006). "Resource and Capability Constraints to Innovation in Small and Large Plants". Small Bussiness Economics, 26, pp. 257-277.

LEVINSOHN, J. y PETRIN, A. (2003). "Estimating production functions using inputs to control for unobservables". Review of Economic Studies, 70(2), pp. 317-342.

LILEEVA, A. y TREFLER, D. (2010). "Improved Access to Foreign Markets Raises PlantLevel Productivity...For Some Plants". Quarterly Journal of Economics, 125(3), pp. 1051-1099.

MELITZ, M.J. (2003). "The impact of trade on intra-industry reallocations and aggregate industry productivity". Econometrica, 71, pp. 1695-1725.

MELITZ, M.J. y OTTAVIANO, G.I.P. (2008). "Market Size, Trade, and Productivity". Review of Economic Studies, 75(1), pp. 295-316.

MUÑOZ-SEPÚLVEDA, J.A. (2014). "Residual exports and domestic demand: an empirical analysis". MPRA Working Papers 54799. https://mpra.ub.uni-muenchen.de/ 54799/1/MPRA_paper_54799.pdf. [Último acceso: Septiembre de 2016].

OUM, S.; NARJOKO, D. y HARVIE, C. (2014). "Constraints, Determinants of SME Innovation, and the Role of Government Support". ERIA Discussion Paper Series no. 
2014-10. http://www.eria.org/ERIA-DP-2014-10.pdf. [Último acceso: Septiembre de 2016].

SMOLNY, W. (2003). "Determinants of innovation behavior and investment estimates for west-german manufacturing firms". Economics of Innovation and New Technology, 12(5), pp. 449-463.

SODERBERY, A. (2014). "Market size, structure, and access: Trade dynamics with capacity constraints". European Economic Review, 70, pp. 276-298. 


\section{Apéndice}

Tabla A.1.

Estadísticos descriptivos de las variables

\begin{tabular}{llcccc}
\hline Variable & Nombre & Media & $\begin{array}{c}\text { Desviación } \\
\text { Estándar }\end{array}$ & Mínimo & Máximo \\
\hline Exportación & $X$ & 0,62 & 0,49 & 0 & 1 \\
Inversión I+D & $I+D$ & 0,35 & 0,48 & 0 & 1 \\
Utilización Capacidad Productiva & $U$ & 0,80 & 0,16 & 0 & 1 \\
Tamaño (número empleados) & - & 227,64 & 663,62 & 1 & 15003 \\
Productividad Total de los Factores (log) & PTF & 5,03 & 0,60 & $-1,89$ & 7,85 \\
Estructura Propiedad & Propiedad & 0,20 & 0,40 & 0 & 1 \\
Mercado Recesivo & Recesivo & 0,33 & 0,47 & 0 & 1 \\
Mercado Expansivo & Expansivo & 027 & 0,45 & 0 & 1 \\
\hline
\end{tabular}

Fuente: Elaboración propia a partir de los datos de la ESEE. 
